\title{
A EFETIVIDADE DA TERAPIA MANUAL NO TRATAMENTO DE DISFUNÇÕES TEMPOROMANDIBULARES (DTM): UMA REVISÃO DA LITERATURA
}

\author{
THE EFFECTIVENESS OF MANUAL THERAPY IN THE TREATMENT OF TEMPOROMANDIBULAR \\ DISORDERS (TMD): A REVIEW OF THE LITERATURE
}

\author{
Lúcia de Fátima da Silva Santos ${ }^{a}$, Mayane Carneiro Alves Pereirab \\ alucia3584@hotmail.com, bmayanealves@hotmail.com \\ Universidade Federal do Piauí - Teresina (PI), Brasil
}

Data de recebimento do artigo: 16/09/2015

Data de aceite do artigo: 04/03/2016

\section{RESUMO}

Introduçáo: A disfunção temporomandibular (DTM) é uma subclassificação das disfunçôes musculoesqueléticas e apresenta etiologia indefinida. Seus sintomas mais comuns são a dor orofacial e a diminuiçáo da mobilidade articular da boca, sensaçôes que reduzem a qualidade de vida de seu portador. A fisioterapia surge como um importante método terapêutico, e entre os métodos comumente utilizados existe a terapia manual. Objetivo: Realizar uma revisão da literatura sobre a efetividade das condutas terapêuticas manuais no tratamento da DTM. Materiais e métodos: Realizou-se uma busca nas bases eletrônicas de dados Lilacs, SciELO, Medline e Pubmed, reunindo ensaios clínicos do período de 2004 a 2014 que documentassem as condutas terapêuticas manuais, isoladas ou associadas, utilizadas no tratamento da DTM em adultos. Resultados: Foram revisados onze artigos, referentes à intervenção da terapia manual na DTM, dos quais nove são nacionais e dois internacionais. Os estudos apontaram eficácia da terapia manual no alívio da sintomatologia dolorosa, melhora do padrão de contração da musculatura mastigatória e restauração da mobilidade articular através dos métodos utilizados no tratamento, e relataram que a significância do resultado foi obtida a partir da associação das técnicas manuais a outras terapias, como recursos eletrofísicos e drogas. Conclusóes: A partir do estudo, é possível observar que a terapia manual apresenta resultados satisfatórios no tratamento de pacientes com DTM e que a associação da terapia manual com outros recursos terapêuticos potencializa os efeitos obtidos no tratamento desses pacientes, apresentando resultados mais significativos.

Palavras-chave: Articulação temporomandibular; síndrome da disfunção temporomandibular; transtornos da articulação temporomandibular; fisioterapia.

\section{ABSTRACT}

Introduction: Temporomandibular disorder (TMD) is a subclassification of musculoskeletal dysfunctions and have unknown etiology. Its most common symptoms are orofacial pain and decreased joint mobility of the mouth, sensations that reduce the quality of life of its bearer. Physical therapy appears as an important therapeutic method, and among the methods commonly used, exist the manual therapy. Objective: To conduct a literature review on the effectiveness of manual therapeutic approaches in the treatment of TMD. Materials and methods: We conducted a search in the electronic databases of data Lilacs, SciELO, Medline, and Pubmed gathering clinical trials from 2004 to 2014 which documented manual therapeutic conducts, isolated or associated, used for treating TMD in adults. Results: Eleven articles referring to the intervention of manual therapy in TMD were reviewed, of which nine are Brazilian and two are international. The studies showed effectiveness of manual therapy in relieving painful symptoms, improvement of the pattern of contraction of the masticatory musculature, and restoration of joint mobility through the methods used in the treatment and reported that the significance of the results was obtained from the association between manual techniques and others therapies, such as electrophysical resources and drugs. Conclusion: Based on the study, one can observe that manual therapy presents satisfactory results in treating patients with TMD and that the combination of manual therapy with other therapeutic resources enhances the effects obtained in the treatment of these patients with more significant results.

Keywords: Temporomandibular joint; temporomandibular joint dysfunction syndrome; temporomandibular joint disorders; physiotherapy. 


\section{Introdução}

Os distúrbios ou desordens da articulação temporomandibular (DTM) são uma patologia clínica caracterizada por uma série de sinais e sintomas ${ }^{1}$ que afetam $86 \%$ da população ${ }^{2}$, cujos principais sinais clínicos são considerados a dor orofacial e a restrição de mobilidade articular da boca e da coluna cervical.

A DTM é considerada uma subclassificação das disfunçôes musculoesqueléticas e comumente apresenta-se de forma recorrente ou crônica, com uma substancial flutuaçáo de sinais e sintomas ao longo do tempo ${ }^{3}$, sendo mais frequente no sexo feminino ${ }^{4,5} \mathrm{e}$ em indivíduos entre 20 e 40 anos $^{6,7}$.

A tentativa de isolar uma causa nítida e universal da DTM não tem sido bem-sucedida. Estudos recentes concluem que ela tem origem multifatorial. Devido à sua etiologia indefinida e ao seu caráter autolimitante, para o tratamento inicial é recomendada a utilização de terapias não invasivas e reversíveis, a fim de amenizar cargas adversas, que perpetuam o problema, controlar a dor, recuperar a função do aparelho mastigatório e reeducar o paciente ${ }^{8}$.

Baseando-se nessas premissas, o seu tratamento requer, muitas vezes, a participaçáo de diversos profissionais da área da saúde, tais como cirurgião-dentista, fisioterapeuta, fonoaudiólogo, psicólogo, neurologista, reumatologista e cirurgiáo buco-maxilo-facial ${ }^{1}$.

As técnicas fisioterapêuticas utilizadas nos quadros de DTM constituem um modelo importante de terapia coadjuvante dentro de um arsenal terapêutico orientado a solucionar diretamente os problemas. O tratamento fisioterapêutico é direcionado para o alívio da dor da musculatura envolvida, a reeducação do sistema neuromuscular, o restabelecimento da posição de repouso mandibular, a recuperação da coordenação muscular ${ }^{9}$, a resolução dos sintomas, o reequilíbrio muscular e a restauração da função comprometida, obtendo resultados mais duradouros ${ }^{10}$.

Entre os recursos mais empregados na fisioterapia para o manuseio das DTM estão a termoterapia (calor e frio), os agentes eletromagnéticos (diatermia por ondas curtas e laser de baixa intensidade), o ultrassom terapêutico (UST), a estimulação eletroneural transcutânea (TENS), a terapia manual (massagem, mobilização manual, manipulação e exercícios) ${ }^{10} \mathrm{e}$ a mecanoterapia ${ }^{11}$.

De acordo com Grossi e Chaves ${ }^{12}$, a terapia manual é um recurso fisioterapêutico importante para o tratamento de pacientes com DTM, e a associação de técnicas manuais com outros recursos terapêuticos constitui um modelo importante de intervenção no tratamento dessas desordens.

A terapia manual tem como objetivos, por meio de técnicas de manipulação e de mobilização e de exercícios específicos, estimular a propriocepção e o líquido sinovial, produzir elasticidade a fibras aderidas e reduzir a dor ${ }^{13}$.

Considerando os dados expostos, o presente estudo teve como objetivo realizar uma revisão da literatura sobre a efetividade das técnicas terapêuticas manuais como recurso fisioterapêutico isolado ou associado a outros recursos no tratamento da DTM.

\section{Desenvolvimento}

Este trabalho consiste numa revisão da literatura, baseando-se na consulta às bases de dados eletrônicas Literatura Latino-Americana e do Caribe em Ciências da Saúde (Lilacs), Scientific Electronic Library Online (SciELO) e Medical Literature Analysis and Retrieval Sistem Online (Medline/Pubmed), durante os meses de dezembro de 2014 e fevereiro de 2015. Os termos DeCS (descritores em ciências da saúde) utilizados para a pesquisa foram "temporomandibular joint", "temporomandibular joint dysfunction syndrome", "temporomandibular joint disorders" e "physiotherapy", isolados e com suas combinaçóes. Os descritores foram combinados utilizando os operadores booleanos.

Os artigos identificados pela estratégia de busca foram avaliados inicialmente a partir da leitura dos títulos e resumos, e posteriormente a partir da leitura do artigo na íntegra, utilizando como critérios de inclusão nesta revisão ensaios clínicos publicados no período de 2004 a 2014 que documentassem a aplicação da terapia manual (com ênfase em massoterapia, liberação miofascial e manipulações musculoesqueléticas) no tratamento das DTM em adultos, nos idiomas português e inglês. Foram excluídos os estudos de revisão bibliográfica e revisão sistemática.

Após a supressão das duplicatas, dos artigos que não obedeciam aos critérios de inclusão e daqueles cuja temática não contemplava a abordagem deste estudo, foram incluídos no total onze ensaios clínicos para análise qualitativa dos dados, dos quais nove são nacionais s,14-21 $^{2}$ e dois internacionais ${ }^{22,23}$. Os estudos investigaram um total de 394 pacientes com DTM que foram tratados com técnicas manuais isoladas ou associadas a outros recursos terapêuticos (eletrotermoterapia, terapia medicamentosa e placas oclusais). A quantidade de intervençôes realizadas variou de uma a doze.

Quanto à composição das amostras, quatro estudos $^{15,16,19,20}$ tiveram amostra contendo apenas mulheres, e sete estudos ${ }^{3,14,17,18,21-23}$ tiveram amostra contendo homens e mulheres. Quanto à faixa etária, os estudos abordaram indivíduos com idade entre 18 e 69 $\operatorname{anos}^{3,14-23}$. Os dados dos artigos obtidos estáo expostos na Tabela 1. 
Tabela 1: Características dos ensaios clínicos selecionados.

\begin{tabular}{|c|c|c|c|c|c|}
\hline $\begin{array}{l}\text { Autor/ } \\
\text { Ano }\end{array}$ & $\begin{array}{l}\text { Tipo de } \\
\text { Estudo }\end{array}$ & $\begin{array}{l}\text { Período do } \\
\text { estudo/ } \\
\text { No de atend. }\end{array}$ & Amostra & Intervençóes & Resultados \\
\hline $\begin{array}{l}\text { Capellini et al., } \\
2006\end{array}$ & $\begin{array}{l}\text { Ensaio } \\
\text { clínico }\end{array}$ & $\begin{array}{c}3 \text { semanas/ } 15 \\
\text { atend. }\end{array}$ & $\begin{array}{l}10 \text { mulheres e } 2 \text { homens. } \\
\text { G1: } 1 \mathrm{H}, 5 \mathrm{M} . \\
\text { G2: } 1 \mathrm{H}, 5 \mathrm{M} . \\
19 \text { a } 22 \text { anos. }\end{array}$ & $\begin{array}{l}\text { G1: massagem na face e no } \\
\text { pescoço; } 4 \text { sessōes EMG. } \\
\text { G2: } 4 \text { sessōes EMG. }\end{array}$ & $\begin{array}{l}\text { Redução significativa na dor } \\
\text { em G1. }\end{array}$ \\
\hline $\begin{array}{l}\text { Castro et al., } \\
2006\end{array}$ & $\begin{array}{l}\text { Ensaio } \\
\text { clínico } \\
\text { randomizado }\end{array}$ & $\begin{array}{l}\text { Maio a novem- } \\
\text { bro de } 2004 / 6 \\
\text { atend. }\end{array}$ & $\begin{array}{l}12 \text { mulheres com DTM. } \\
\text { G1: } 6 . \\
\text { G2: } 6 . \\
18 \text { a } 52 \text { anos. }\end{array}$ & $\begin{array}{l}\text { G1: TENS; alongamento. } \\
\text { G2: TENS; alongamento; te- } \\
\text { rapia de liberaçáo posicional. }\end{array}$ & $\begin{array}{c}\text { G2: redução significativa da } \\
\text { dor e aumento significativo } \\
\text { da ADM de protrusão, flexão, } \\
\text { extensão e rotação cervical. G1: } \\
\text { sem mudança. }\end{array}$ \\
\hline $\begin{array}{l}\text { George et al., } \\
2007\end{array}$ & $\begin{array}{l}\text { Ensaio } \\
\text { clínico }\end{array}$ & 1 atend. & $\begin{array}{c}64 \text { mulheres e } 37 \text { homens. } \\
\text { G1: } 34 . \\
\text { G2: } 34 . \\
\text { G3: } 33 . \\
\text { 24,6 } 62,6 \text { anos. }\end{array}$ & $\begin{array}{l}\text { G1: Técnica de liberação } \\
\text { miofascial nos músculos } \\
\text { suboccipitais. } \\
\text { G2: Manipulação thrust de } \\
\text { alta velocidade baixa ampli- } \\
\text { tude (HVLA) em C1. } \\
\text { G3: Grupo controle. }\end{array}$ & $\begin{array}{l}\text { Não houve diferença significati- } \\
\text { va na ADM de abertura da boca } \\
\text { entre os grupos. }\end{array}$ \\
\hline $\begin{array}{l}\text { Tosato et al., } \\
2007\end{array}$ & $\begin{array}{l}\text { Ensaio } \\
\text { clínico } \\
\text { randomizado }\end{array}$ & 1 atend. & $\begin{array}{c}20 \text { mulheres com DTM. } \\
\text { G1: } 10 . \\
\text { G2: } 10 . \\
22 \text { a } 46 \text { anos. }\end{array}$ & $\begin{array}{c}\text { G1: Massoterapia na face } \\
\text { durante } 30 \text { minutos. } \\
\text { G2: TENS durante } 30 \\
\text { minutos. }\end{array}$ & $\begin{array}{l}\text { G1 e G2 apresentaram melhora } \\
\text { no padráo de contração muscu- } \\
\text { lar e na reduçáo da dor. }\end{array}$ \\
\hline $\begin{array}{c}\text { Andrade; Frare, } \\
2008\end{array}$ & $\begin{array}{l}\text { Ensaio } \\
\text { clínico } \\
\text { randomizado }\end{array}$ & $\begin{array}{l}\text { Maio a agosto de } \\
2007 / 12 \text { atend. }\end{array}$ & $\begin{array}{l}20 \text { pacientes. } \\
\text { G1: } 7 \mathrm{M}, 3 \mathrm{H} \text {. } \\
\text { G2: } 8 \mathrm{M}, 2 \mathrm{H} \text {. } \\
18 \text { a } 40 \text { anos. }\end{array}$ & $\begin{array}{c}\text { G1: Técnica de terapia } \\
\text { manual. } \\
\text { G2: Técnica de terapia } \\
\text { manual; laserterapia de baixa } \\
\text { potência. }\end{array}$ & $\begin{array}{c}\text { G2 apresentou resultados mais } \\
\text { significativos para a reduçáo da } \\
\text { dor. }\end{array}$ \\
\hline $\begin{array}{l}\text { Cuccia et al., } \\
2010\end{array}$ & $\begin{array}{l}\text { Ensaio } \\
\text { clínico } \\
\text { randomizado }\end{array}$ & $\begin{array}{l}\text { Setembro de } \\
2008 \text { a fevereiro } \\
\text { de } 2009 .\end{array}$ & $\begin{array}{c}50 \text { pacientes. } \\
\text { G1:12 } \mathrm{H}, 13 \mathrm{M} . \\
\mathrm{G} 2: 10 \mathrm{H} \\
15 \mathrm{M} . \\
18 \text { a } 50 \text { anos. }\end{array}$ & $\begin{array}{l}\text { G1: liberação miofascial; } \\
\text { energia muscular; técni- } \\
\text { cas de osteopatia; terapia } \\
\text { crânio-sacral. } \\
\text { G2: alongamento; relaxamen- } \\
\text { to; compressas quentes e/ou } \\
\text { frias; TENS. }\end{array}$ & $\begin{array}{l}\text { G1 e G2 melhoraram durante o } \\
\text { tratamento. } \\
\text { G1 necessitou significativamen- } \\
\text { te de menos medicaçáo. }\end{array}$ \\
\hline $\begin{array}{l}\text { Kamon-seki } \\
\text { et al., } 2012\end{array}$ & $\begin{array}{l}\text { Ensaio } \\
\text { clínico } \\
\text { randomizado }\end{array}$ & 1 atend. & $\begin{array}{c}29 \text { homens e } 54 \text { mulheres. } \\
\text { G1: } 41 . \\
\text { G2: } 42 . \\
18 \text { a } 35 \text { anos. }\end{array}$ & $\begin{array}{l}\text { G1: manipulação thrust de } \\
\text { alta velocidade e baixa ampli- } \\
\text { tude (HVLA). } \\
\text { G2: intervençáo simulada. }\end{array}$ & $\begin{array}{l}\text { A HVLA promoveu aumento } \\
\text { na abertura da boca em sujeitos } \\
\text { sintomáticos imediatamente } \\
\text { após a intervençáo. }\end{array}$ \\
\hline $\begin{array}{l}\text { Torres et al., } \\
2012\end{array}$ & $\begin{array}{l}\text { Ensaio } \\
\text { clínico }\end{array}$ & $\begin{array}{l}\text { Três semanas/ } 10 \\
\text { atend. }\end{array}$ & $\begin{array}{c}10 \text { pacientes. } \\
\text { G1: } 5 . \\
\text { G2: } 5 . \\
\text { Idade média de } 34 \text { anos. }\end{array}$ & $\begin{array}{l}\text { G1: TENS; ultrassom; } \\
\text { massagem; alongamento; } \\
\text { relaxamento cervical. } \\
\text { G2: medicamentos; placas } \\
\text { miorrelaxantes. }\end{array}$ & $\begin{array}{l}\text { G1 e G2 apresentaram alívio } \\
\text { da dor. } \\
\text { A fisioterapia foi indicada para } \\
\text { aliviar a dor em curto prazo. }\end{array}$ \\
\hline $\begin{array}{l}\text { Dias et al., } \\
2013\end{array}$ & $\begin{array}{l}\text { Ensaio } \\
\text { clínico }\end{array}$ & 4 semanas. & $\begin{array}{l}7 \text { mulheres. } \\
19 \text { a } 69 \text { anos. }\end{array}$ & $\begin{array}{c}\text { Técnica de inibição muscular } \\
\text { ativa. }\end{array}$ & $\begin{array}{l}\text { Observado aumento da ADM } \\
\text { na abertura da boca e na força da } \\
\text { mordida. }\end{array}$ \\
\hline $\begin{array}{l}\text { Nascimento } \\
\text { et al., } 2013\end{array}$ & $\begin{array}{l}\text { Ensaio } \\
\text { clínico } \\
\text { randomizado }\end{array}$ & 8 semanas. & $\begin{array}{l}20 \text { mulheres. } \\
\text { G1: } 10 . \\
\text { G2: } 10 . \\
25 \text { a } 56 \text { anos. }\end{array}$ & $\begin{array}{l}\text { G1: } 8 \text { bloqueios anestésicos } \\
\text { do nervo auriculotemporal. } \\
\text { G2: bloqueio anestésico; } \\
\text { fisioterapia. }\end{array}$ & $\begin{array}{c}\text { G1 e G2 apresentaram melhora } \\
\text { da dor e da ADM da boca e } \\
\text { cervical. } \\
\text { G1 apresentou resultados mais } \\
\text { significativos. }\end{array}$ \\
\hline $\begin{array}{l}\text { Gomes et al., } \\
2014\end{array}$ & $\begin{array}{l}\text { Ensaio } \\
\text { clínico } \\
\text { randomizado }\end{array}$ & $\begin{array}{l}4 \text { semanas/ } 3 \\
\text { atend. }\end{array}$ & $\begin{array}{c}10 \text { homens e } 49 \text { mulheres. } \\
\text { G1: } 2 \mathrm{H}, 13 \mathrm{M} . \\
\text { G2: } 3 \mathrm{H}, 12 \mathrm{M} . \\
\text { G3: } 1 \mathrm{H}, 14 \mathrm{M} . \\
\text { G4: } 4 \mathrm{H}, 10 \mathrm{M} . \\
18 \text { a } 40 \text { anos. }\end{array}$ & $\begin{array}{c}\text { G1: massagem nos músculos } \\
\text { masseter e temporal anterior, } \\
\text { bilateralmente. } \\
\text { G2: uso de placa oclusal por } \\
\text { quatro semanas. } \\
\text { G3: tratamento combinado } \\
\text { (massagem e placa oclusal). } \\
\text { G:4 tratamento com placa de } \\
\text { silicone. }\end{array}$ & $\begin{array}{l}\text { G1 e G2 não obtiveram mudan- } \\
\text { ça na atividade eletromiográ- } \\
\text { fica dos músculos masseter ou } \\
\text { temporal. } \\
\text { Combinação de terapias provo- } \\
\text { cou uma redução na intensidade } \\
\text { de sinais e sintomas entre os } \\
\text { indivíduos com DTM severa e } \\
\text { bruxismo. }\end{array}$ \\
\hline
\end{tabular}

Legenda: DTM = Disfunção ou Distúrbio Temporomandibular; TENS = Estimulaçấo Elétrica Nervosa Transcutânea; EVA = Escala Visual Analógica; $\mathrm{EMG}=$ Eletromiografia; $\mathrm{ATEND}=$ Atendimento; $\mathrm{ADM}=$ Amplitude de Movimento. 
Neste estudo buscou-se esclarecer a eficácia das técnicas manuais no tratamento de pacientes com DTM, e os resultados encontrados apontam que a técnica é eficaz quando aplicada isoladamente e quando associada a outros recursos terapêuticos, corroborando a importância da fisioterapia no tratamento das disfunçóes da $\mathrm{ATM}^{24}$, bem como a efetividade das técnicas de terapia manual.

\section{A DTM e a dor}

Os estudos revisados mostraram, quanto à composição das amostras, a prevalência do gênero feminino, semelhante aos dados registrados por outros pesquisadores $^{4,5}$, que relatam que as mulheres possuem mais sintomas e maior predisposição para DTM que os homens ${ }^{25}$. A faixa etária encontrada neste estudo variou entre os vinte e os sessenta anos de vida, concordando com os dados encontrados em outros estudos ${ }^{6,7}$ e com as diretrizes apontadas pela Academia Americana de Dor Orofacial, que preconizou a prevalência de DTM entre os vinte e os quarenta anos de vida ${ }^{10,18}$.

Esta revisão concordou com outros estudos ${ }^{26-28}$, segundo os quais a terapia manual apresenta efeitos significativos na disfunção temporomandibular, inclusive nos principais sinais clínicos, proporcionando elevação do limiar de dor, melhora do padrão de contração da musculatura mastigatória, melhora da atividade elétrica dos músculos tratados e redução da sensibilidade dolorosa dos músculos a palpação e restauração da mobilidade articular.

A dor muscular é a principal queixa relatada por pacientes com DTM e ocorre associada à sensação de fadiga e tensão muscular, variando de um leve incômodo ao desconforto extremo, que piora com o uso da musculatura envolvida ${ }^{6}$.

Uma explicaçâo possível para a analgesia induzida manualmente pode estar relacionada à redução da sensibilidade dos mecanoceptores, levando à inibição da dor e proporcionando um período de analgesia imediato, que envolve um processo neurológico chamado bloqueio sensorial ou teoria das comportas. De acordo com essa teoria, o sistema nervoso possui mecanismos capazes de regular o fluxo de impulsos dos nervos periféricos para o sistema nervoso central. Esses mecanismos atuam como um bloqueio para as informações sensoriais, pois fibras nervosas de maior diâmetro (com maior velocidade de condução), como os mecanoceptores, bloqueiam a comporta e contribuem para o alívio da dor, enquanto fibras de pequeno diâmetro abrem a comporta, aumentando a sensação de dor ${ }^{29-31}$.

\section{A terapia manual como resposta}

As técnicas de terapia manual, uma vez que tratam a dor e interferem em propriedades dos tecidos, tais como a elasticidade, possibilitam motivar a capacidade de reparo do organismo, causando reaçóes que levam ao relaxamento da musculatura e à melhora na amplitude de movimento ${ }^{3}$.

Ademais, o alívio da dor e o relaxamento muscular promovido pelas técnicas manuais provocam o restabelecimento da função muscular, possibilitando um aumento significativo da atividade eletromiográfica e uma consequente melhora do padrão de contração muscular, sugerindo que a presença de dor pode levar ao menor recrutamento de unidades motoras, reduzindo capacidade de contração muscular ${ }^{16}$.

Estudos ${ }^{10,23}$ analisados mostram que pacientes tratados com terapia manual isolada ou associada a outros recursos (eletrotermoterapia, cinesioterapia e medicaçóes) permaneceram com melhora da sintomatologia da DTM durante o período de acompanhamento, corroborando os achados de Nicolakis et al..$^{32}$, que estudaram dezessete pacientes na tentativa de avaliar o efeito em longo prazo da terapia manual associada a outras técnicas, e observou que onze permaneceram sem dor e treze foram tratados com sucesso, uma vez que náo precisaram de tratamento nos três anos após a cessaçáo da terapia, concluindo que a terapia manual associada a outras técnicas é uma ferramenta eficaz, a curto e longo prazo, para o tratamento das disfunçóes temporomandibulares. Evidências apontam que a analgesia induzida pela terapia manual ocorre rapidamente após a manipulação, e, com a repetição do tratamento, o efeito analgésico é cumulativo ${ }^{33}$.

As limitações observadas na construção desta revisão foram o tratamento diversificado dos dados e a amostra reduzida dos estudos analisados. Porém, tais dificuldades não inviabilizaram a realização da pesquisa.

\section{Conclusões}

Os resultados deste estudo demonstraram que a terapia manual apresenta efeitos satisfatórios no tratamento de pacientes com DTM e que a associação da terapia manual com outros recursos terapêuticos potencializa esses efeitos. Desse modo, recomenda-se a utilizaçáo de recursos associados à terapia manual como método eficaz, não invasivo e de baixo custo no tratamento dessas desordens. Sugere-se, com o objetivo de comprovar cientificamente a efetividade da terapia manual, a realização de outros estudos que possam complementar e confirmar o conhecimento alcançado. 


\section{Referências}

1. Ferreira FV, Ferreira FV, Tabarelli Z, Peroni ABF. Desordens temporomandibulares: uma abordagem fisioterapêutica e odontológica. Stomatos. 2009 jan/jun;15(28):27-37.

2. Bove SRK, Guimarães AS, Smith RL. Caracterização dos pacientes de um ambulatório de disfunção temporomandibular e dor orofacial. Rev Latinoam Enferm. 2005 set/ out;13(5):686-91.

3. Andrade TNC, Frare JC. Estudo comparativo entre os efeitos de técnicas de terapia manual isolada e associadas à laserterapia de baixa potência sobre a dor em pacientes com disfunção temporomandibular. Rev Gaucha Odontol. 2008 $\mathrm{jul} / \mathrm{set}$;56(3):287-95.

4. Donnarumma MDC, Muzilli CA, Ferreira C, Nemr K. Disfunçóes temporomandibulares: sinais, sintomas e abordagem multidisciplinar. Rev CEFAC. 2010;12(5):788-794.

5. Figueiredo VMG, Cavalcanti AL, Farias ABL, Nascimento SR. Prevalência de sinais, sintomas e fatores associados em portadores de disfunção temporomandibular. Acta Scientiarum Health Sciences. 2009;31(2):159-63.

6. Okeson JP. Tratamento das desordens temporomandibulares e oclusão. 4. ed. São Paulo: Artes Médicas; 2000.

7. Tosato JP, Caria PHF. Prevalência de DTM em diferentes faixas etárias. Rev Gaucha Odontol. 2006 jul/ set;54(3):211-24.

8. Carrara SV, Conti PCR, Barbosa JS. Termo do $1^{\circ}$ Consenso em Disfunção Temporomandibular e Dor Orofacial. Dental Press J Orthod. 2010 jun;15(3):114-20.

9. Marzola FT, Marques AP, Marzola C. Contribuiçấo da fisioterapia para a odontologia nas disfunçôes da articulação temporomandibular. Rev Odonto Ciênc. 2002 abri/ jun;17(36):119-34.

10. Matta MAP. Uma proposta de abordagem fisioterapêutica nas desordens da articulação temporomandibular. Dissertação [Mestrado em Ciências Médicas] - Faculdade de Ciências Médicas, Universidade Estadual de Campinas; 2002.

11. Issa JPM, Silva MAMR, Silva AMBR. Recursos fisioterápicos no tratamento das desordens temporomandibulares. Rev Dor. 2005;6(2):567-72.

12. Grossi DB, Chaves TC. Physiotherapeutic treatment for temporomandibular disorders (TMD). J Braz Oral Sci. 2004;3(10):492-7.

13. Kalamir A, Pollard H, Vitello AL, Bonello R. Manual therapy for temporomandibular disorders: a review of literature. J Bodyw Mov Ther. 2007;11(1):84-90.

14. Capellini VK, Souza GS, Faria CRS. Massage therapy in the management of myogenic TMD: a pilot study. J Appl Oral Sci. 2006;14(1):21-6.

15. Castro FM, Gomes RCV, Salomão JR, Abdon APV. A efetividade da terapia de liberação posicional (TLP) em pacientes portadores de disfunção temporomandibular. Rev Odontol Univ Cid São Paulo. 2006;18(1):67-74.
16. Tosato JP, Gonzalez DAB, Caria PHF. Efeito da massoterapia e da estimulação elétrica nervosa transcutânea na dor e atividade eletromiográfica de pacientes com disfunção temporomandibular. Fisioter Pesqui. 2007 maio/ ago;14(2):21-6.

17. Kamonseki DH, Fonseca CL, Souza TP, Zamunér AR, Peixoto BO, Yi LC. Efeito imediato da manipulação thrust aplicada na coluna cervical alta sobre a abertura ativa da boca: ensaio clínico randomizado. J Health Sci Inst. 2012;30(3):277-80.

18. Torres F, Campos LG, Fillipini HF, Weigert KL, Vecchia GFD. Efeitos dos tratamentos fisioterapêutico e odontológico em pacientes com disfunção temporomandibular. Fisioter Mov. 2012 jan/mar;25(1):117-25.

19. Dias LM, Spinato IL, Vasconcelos TB, Rodrigues DV, Macena RHM, Magalhães CBA, et al. Effects of muscle inhibition technique on relief of masticatory pain in patients with temporomandibular disorders: an experimental study. Conscientiae Saúde. 2013 jun;12(2):298-304.

20. Nascimento MM, Vasconcelos BC, Porto GG, Ferdinanda G, Nogueira CM, Raimundo RC. Physical therapy and anesthetic blockage for treating temporomandibular disorders: a clinical trial. Med Oral Patol Oral Cir Bucal. 2013 Jan;18(1):e81-5.

21. Gomes CAFP, Hage YE, Amaral AP, Politti F, BiasottoGonzalez DA. Effects of massage therapy and occlusal splint therapy on electromyographic activity and the intensity of signs and symptoms in individuals with temporomandibular disorder and sleep bruxism: a randomized clinical trial. Chiropr Man Therap. 2014 Dec;22(1):43.

22. George JW, Fennema J, Maddox A, Nessler M, Skaggs C. The effect of cervical spine manual therapy on normal mouth opening in asymptomatic subjects. J Chiropr Med. 2007 Spring;6(4):141-5.

23. Cuccia AM, Caradonna C, Annunziata V, Caradonna D. Osteopathic manual therapy versus conventional conservative therapy in the treatment of temporomandibular disorders: a randomized controlled trial. J Bodyw Mov Ther. 2010 Apr;14(2):179-84.

24. Mourão NLA, Mesquita VT. A importância da fisioterapia no tratamento das disfunçóes da ATM. Ter Man. 2006;4(16):552-7.

25. Warren MP, Fried JL. Temporomandibular disorders and hormones in women. Cells Tissues Organs. 2001;169(3):187-92.

26. Felício CM, Melchior MO, Ferreira CLP, Silva MA. Otologic symptoms of temporomandibular disorder and effect of orofacial myofunctional therapy. Cranio. 2008 Apr;26(2):118-25.

27. Felício CM, Melchior MO, Silva MA. Effects of orofacial myofunctional therapy on temporomandibular disorders. Cranio. 2010 Oct;28(4):249-59.

28. Vasconcelos DA, Maia MRA, Souza MO, Alves JGB, Nascimento JDS, Dantas EHM. Avaliação eletromiográfica 
e clínica do músculo masseter após manipulação quiropráxica. Rev Inspirar. 2013 mar/abr;5(1):1-6.

29. Melzack R, Wall P. Pain mechanisms: a new theory. Science. 1965 nov;150(3699):971-9.

30. Gillespie BR. Assessment and treatment of muscles, fascia, ligaments, and structures. Cranio. 1990 jan;8(1):51-4.

31. Wright A. Hypoalgesia post-manipulative therapy: a review of potential neurophysiological mechanism. Man Ther. 1995 Nov;1(1):11-6.
32. Nicolakis P, Erdogmus CB, Kollmitzer J, Kerschan-Schindl K, Sengstbratl M, Nuhr M, et al. Long-term outcome after treatment of temporomandibular joint osteoarthritis with exercise and manual therapy. Cranio. 2002 Jan;20(1):23

33. Wright A. An evolving understanding of pain relief following Manual Therapy [Paper presented at the proceedings of the 7th Scientific Conference of the IFOMPT; 2000; Perth, Australia].

\section{Como citar este artigo:}

Santos LFS, Pereira MCA. A efetividade da terapia manual no tratamento de Disfunçóes Temporomandibulares (DTM): uma revisão da literatura. Rev. Aten. Saúde. 2016;14(49):72-77. 